\title{
Colpitts Oscillator: Design and Performance Optimization
}

\section{Ankit Rana*}

B. Tech. (ECE), Bharati Vidyapeeth's College of Engineering, A-4, Paschim Vihar, New Delhi, India

\begin{abstract}
From the very fundamental oscillator, a simple pendulum wherein there is a constant energy switch between potential and kinetic energy, oscillators have seen groundbreaking changes in setup, operation and their applicability. There are harmonic oscillators which produce a continuous sine wave output of certain frequencies as per the passive components involved. Additionally, are known Relaxation oscillator which yield triangular, square and sawtooth waves as output to name a few. The present paper deals with the details of how a fundamental Colpitts oscillatory circuit can be designed. Furthermore, we would take a look at optimizing its performance with change in several dependent characteristics in oscillation. We would conclude with an inference pertaining to the best customization with could be put to practical usage.
\end{abstract}

Keywords: Feedback; LC combination; Multisim; Tank circuit; VFO; Resonance; Magnetic energy leakage; Microfarad(uF); Oscillators; Sinusoidal

\section{Introduction}

Back in mid-1912, Edwin Armstrong while carrying out experiments with triodes wasn't aware of a spectacular phenomena due to a component which was going to serve as the basis of coupling and amplification amongst many other applications. Until then, the experiments had incorporated these devices as a detector for amplitude modulated waves. Having utilized them for this functionality, nobody was aware of the reasons as to why that was happening. By means of coupling one terminal of the device to another, it was observed that he could achieve large signal gain. We today term the phenomenon as positive feedback in circuits. He, with his invention of the radio had produced a unique oscillator. The uniqueness was justified since the limitation of other oscillators producing output in the $\mathrm{kHz}$ domain had now been extended to the $\mathrm{MHz}$ domain [1].

Ever since then, there has been a plethora of oscillator circuits that have been invented and employed industrially worldwide. Continuous Sine Wave oscillators or Square wave oscillators find their application in a myriad of fields in converting DC input to a variant A.C. output, in amplification of signals, synchronization purposes to name a few. One such circuit which utilizes storage and dissipation of magnetic energy, namely the Colpitts Oscillator has formed the basis of this study. A few of modifications in the conventional circuit have hereby been inferred.

\section{The Colpitts Oscillator}

\section{Classification}

The Colpitts Oscillator is known to work on feedback from the divider setup that is used in the circuit. The voltage divider is either made by 2 inductors or by using tapping on the single inductor. In either of the cases, if the desired application is that of a VFO, the usage is not as much preferred as with the case of Clapp Oscillator. In the latter, an extra capacitor is used for tuning to the optimum frequency and hence a better sustained waveform is achievable readily $[2,3]$.

However, Colpitts form the basis of either of these circuitries, capacitor-tuned Clapp or the conventional circuit. And consequently, is believed in this experimental study to be encompassing the behavioral results for the counterpart circuits as well [2].

\section{Frequency tuning and parameters that effect it}

Since the Colpitts oscillator is a type of tank circuit (LC combination) and works on feedback of energy, the mathematical expression underlying its operation is the same as that of first-order LC circuit i.e.

$$
f=\left(1 / 2 \pi \sqrt{L^{*} C}\right)
$$

And since, the Colpitts must have two capacitors for compensating purposes, the mathematical expression to obtain the frequency of operation is depicted as $[4,5]$ :

$$
f=1 / 2 \pi \sqrt{\frac{L(C 1 * C 2)}{(C 1+C 2)}}
$$

It can be noted that the two capacitors in series with each other result in the expression in the denominator.

From the study of its parasitic elements and the transconductance $\left(g_{m}\right)$ concept, it is known that a negative value of input resistance only would be able to sustain oscillations at the output $[6,7]$.

Oscillations are obtained only for a large value of transconductance $\left(g_{m}\right)$ and for smaller values of capacitor elements used.

\section{Experimental Approach to Colpitts Oscillator Design}

A industrially acclaimed simulator, namely Multisim was used to observe the influence of several parameters on a Colpitts oscillator design with multiple modifications and optimization aims in mind $[5,7]$.

A first order Colpitts Oscillator has been drawn as a schematic over the simulator. This schematic circuit was introduced with several modifications viz. change in input resistance, changes in capacitors and the inductor coil's inductance. The parameters mentioned herewith are the fundamental governing dependencies in the performance of a Colpitts Oscillator. Increase in the value of any of these would leave

*Corresponding author: Ankit Rana, B. Tech. (ECE), Bharati Vidyapeeth's College of Engineering, A-4, Paschim Vihar, New Delhi-110063, India, E-mail: ankitrana1709@gmail.com

Received September 01, 2014; Accepted September 19, 2014; Published October 01, 2014

Citation: Rana A (2014) Colpitts Oscillator: Design and Performance Optimization J Electr Electron Syst 3: 132. doi:10.4172/2332-0796.1000132

Copyright: (c) 2014 Rana A. This is an open-access article distributed under the terms of the Creative Commons Attribution License, which permits unrestricted use, distribution, and reproduction in any medium, provided the original author and source are credited. 
an impact on the output sinusoid produced. From a perfect sinusoid under optimum conditions (mentioned as conclusion) to a distorted wave output, the tank circuit's myriad of oscillatory behaviors have been incorporated $[8,9]$.
The effects were simulated over Multisim and analyzed over time. Conclusions from the same were drawn (Figures 1-12).

The behavior as per the results obtained from this comparative study have been incorporated as conclusions to the customization activity.

\section{Schematic Colpitts Oscillator}

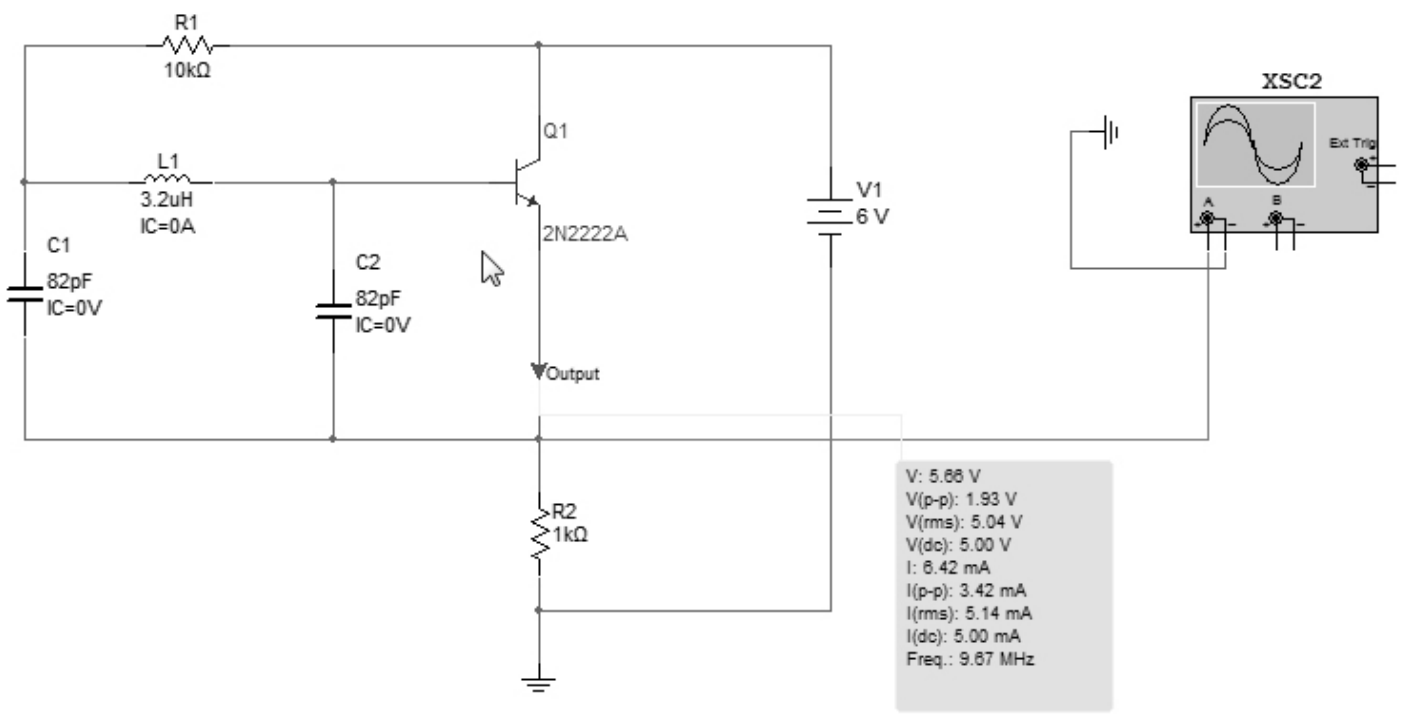

Figure 1: Fundamental design of a Colpitts Oscillator (Multisim view).

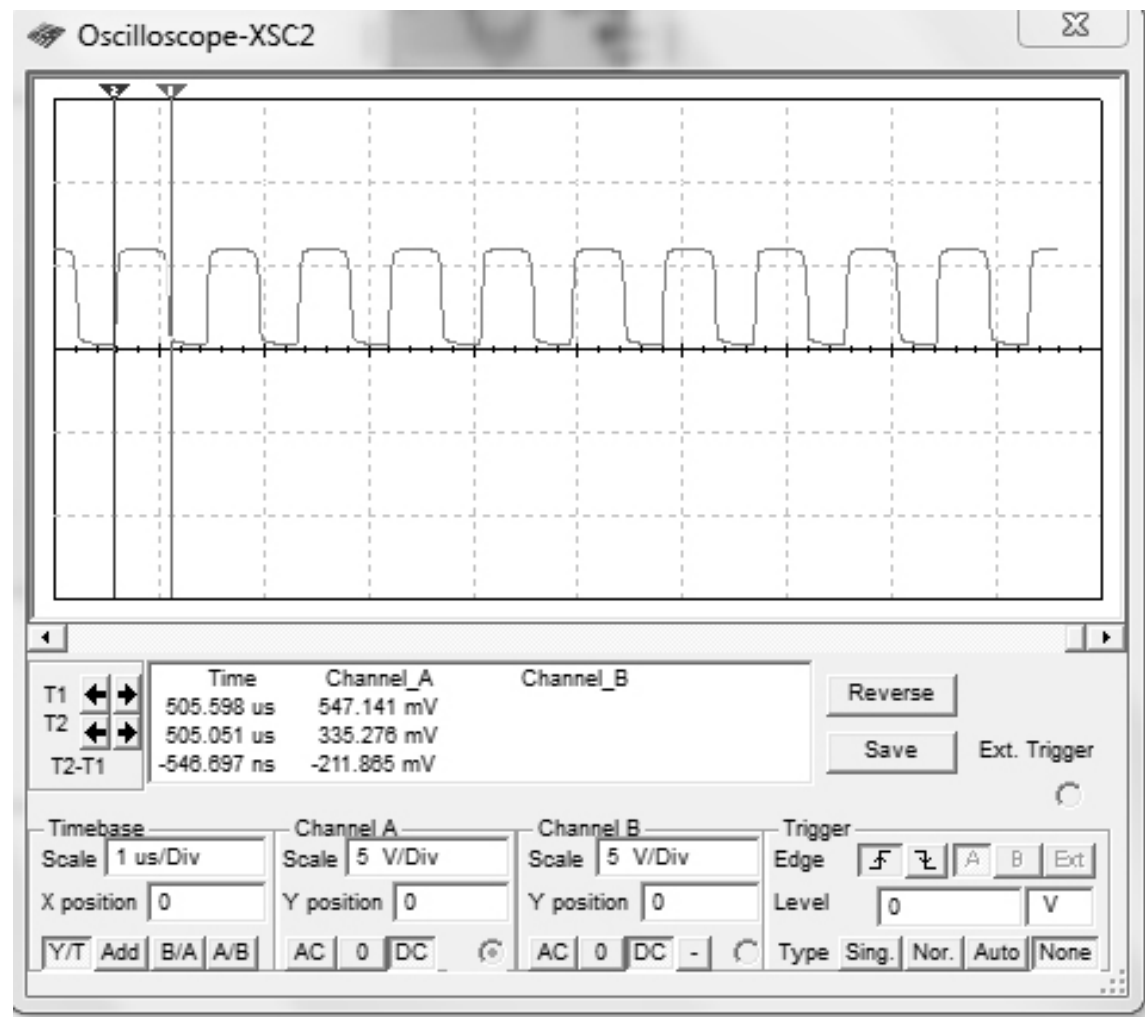

Figure 2: Output waveform for the circuit in figure with inductor coil of $320 u \mathrm{H}$. 
Oscilloscope-XSC2

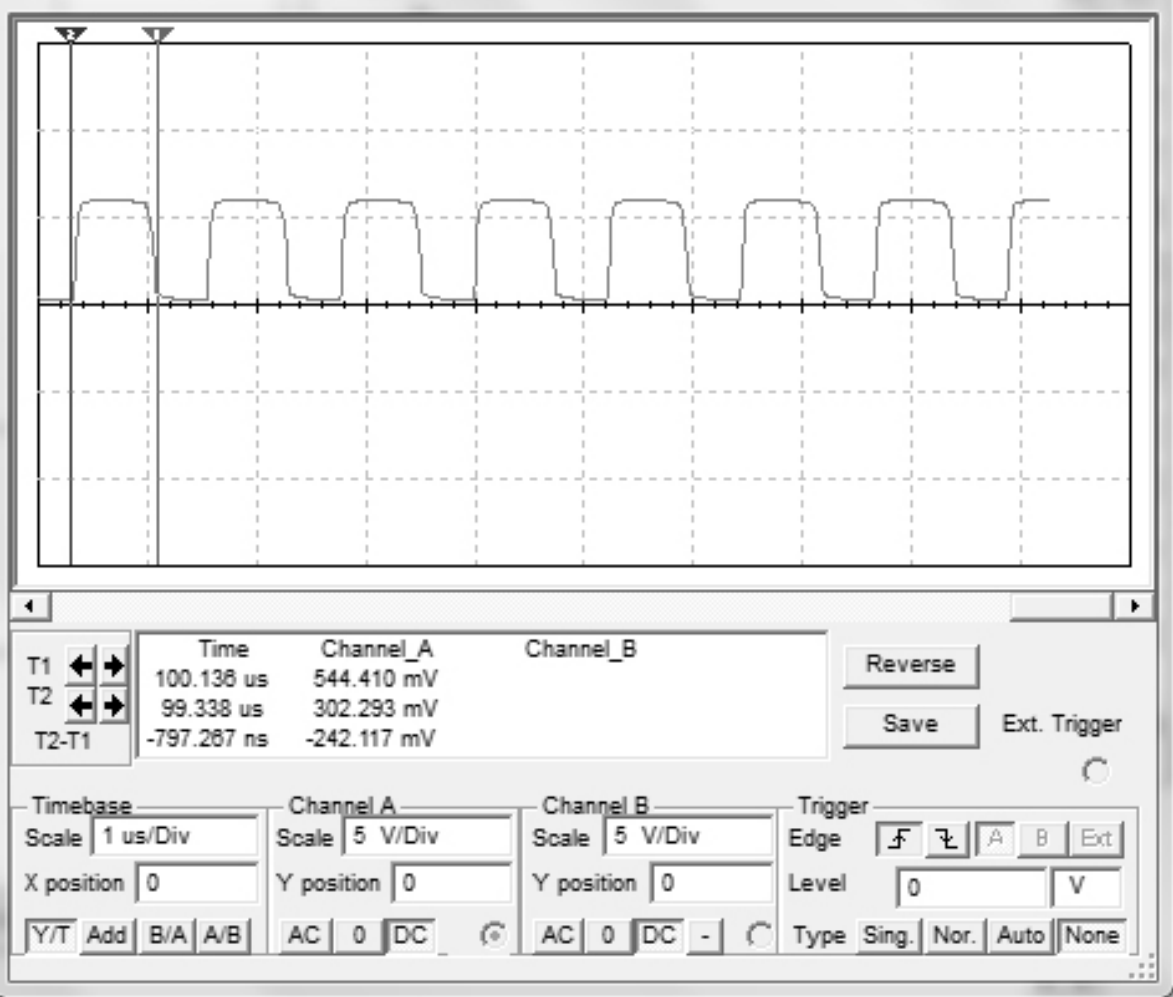

Figure 3: Output waveform for the circuit in figure with inductor coil of $640 \mathrm{uH}$.

\begin{tabular}{|c|c|c|c|}
\hline & & & 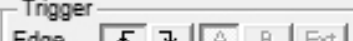 \\
\hline & & UIV & \\
\hline & \begin{tabular}{l|l|l|l|l|}
$A C$ & 0 \\
$D C$
\end{tabular} & \begin{tabular}{l|l}
$A C$ & 0 \\
$D C$
\end{tabular} & \\
\hline
\end{tabular}

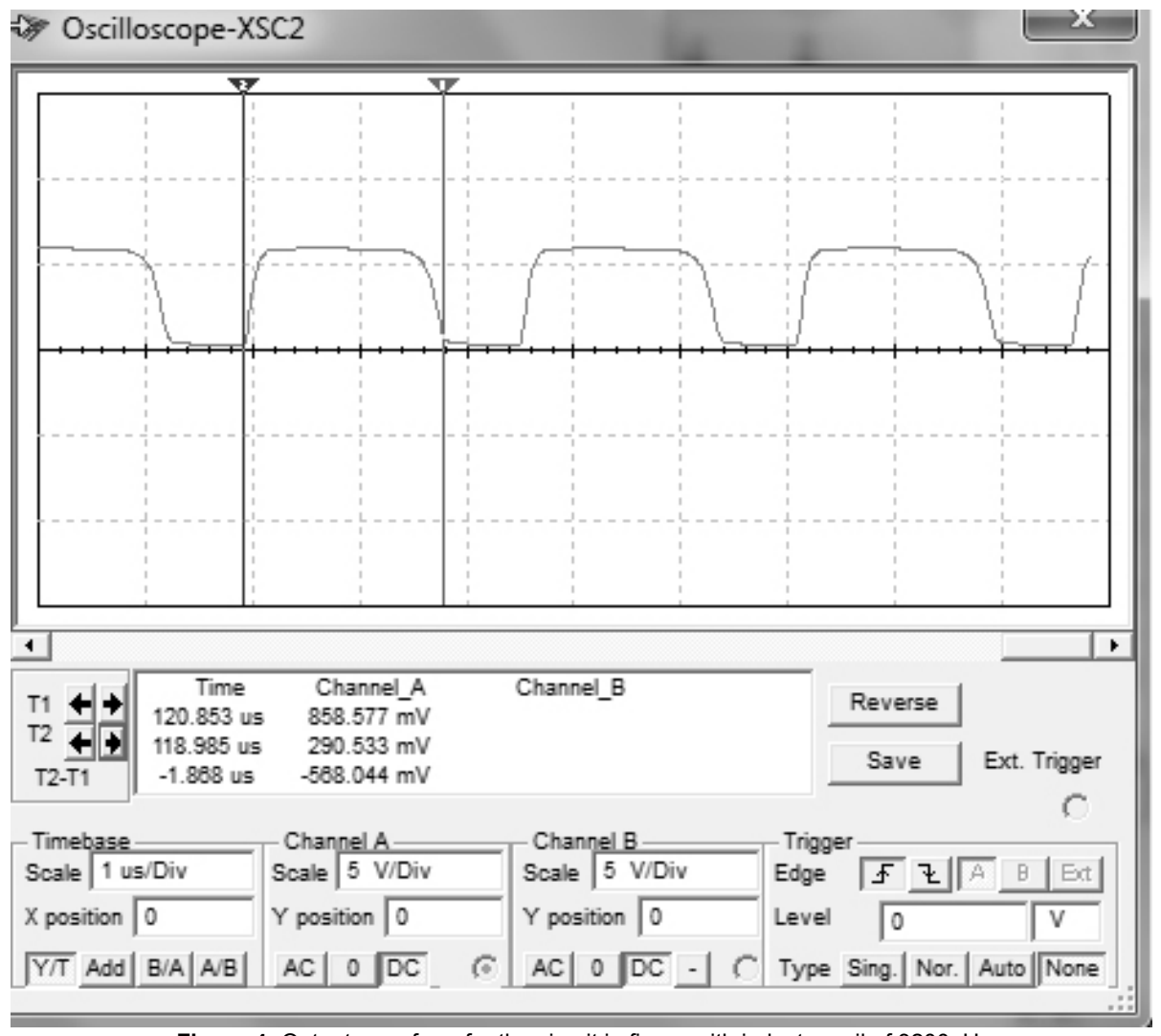

Figure 4: Output waveform for the circuit in figure with inductor coil of 3200uH. 


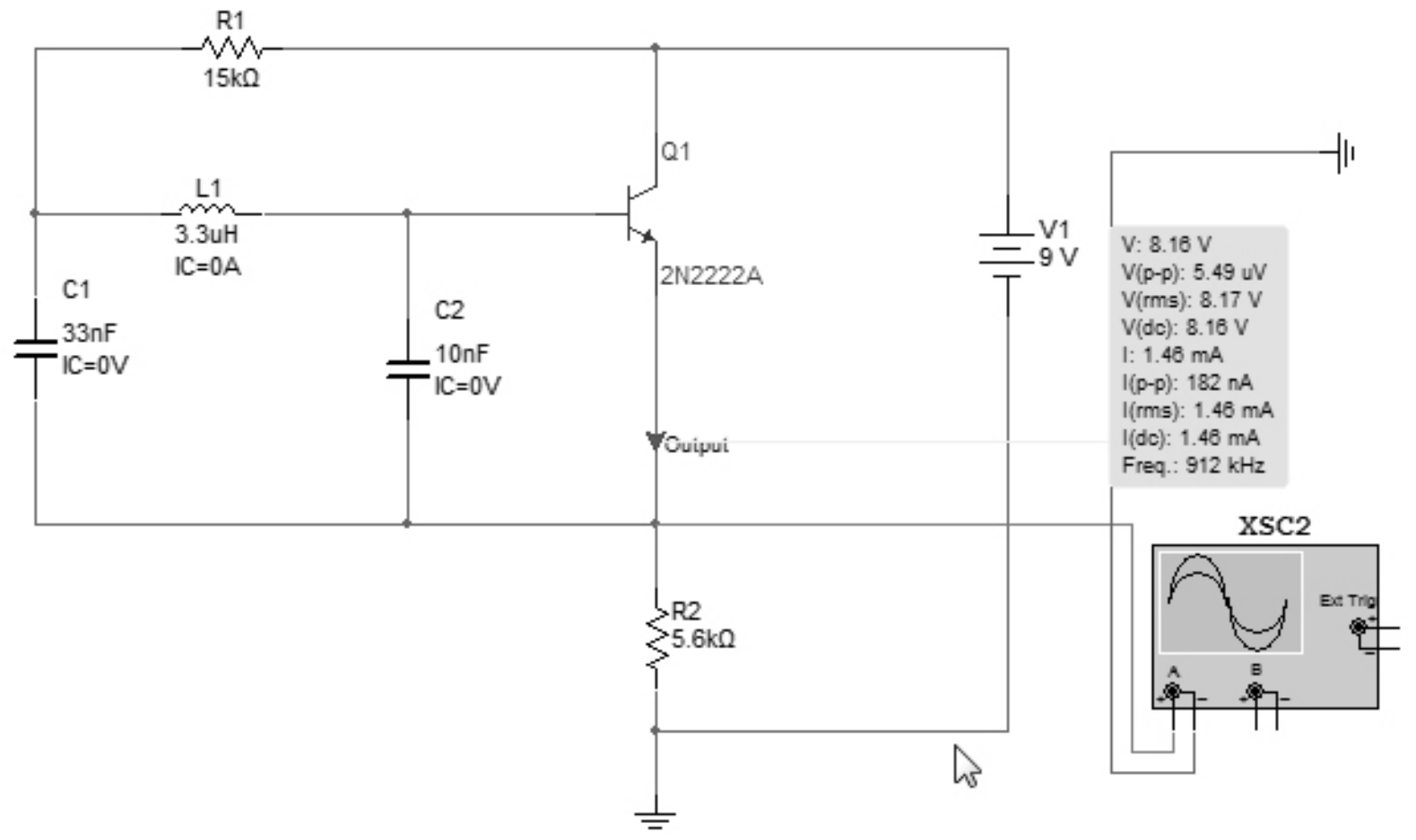

Figure 5: Colpitts Oscillator schematic diagram with unequal capacitors and resistances varied in the simulator design window.

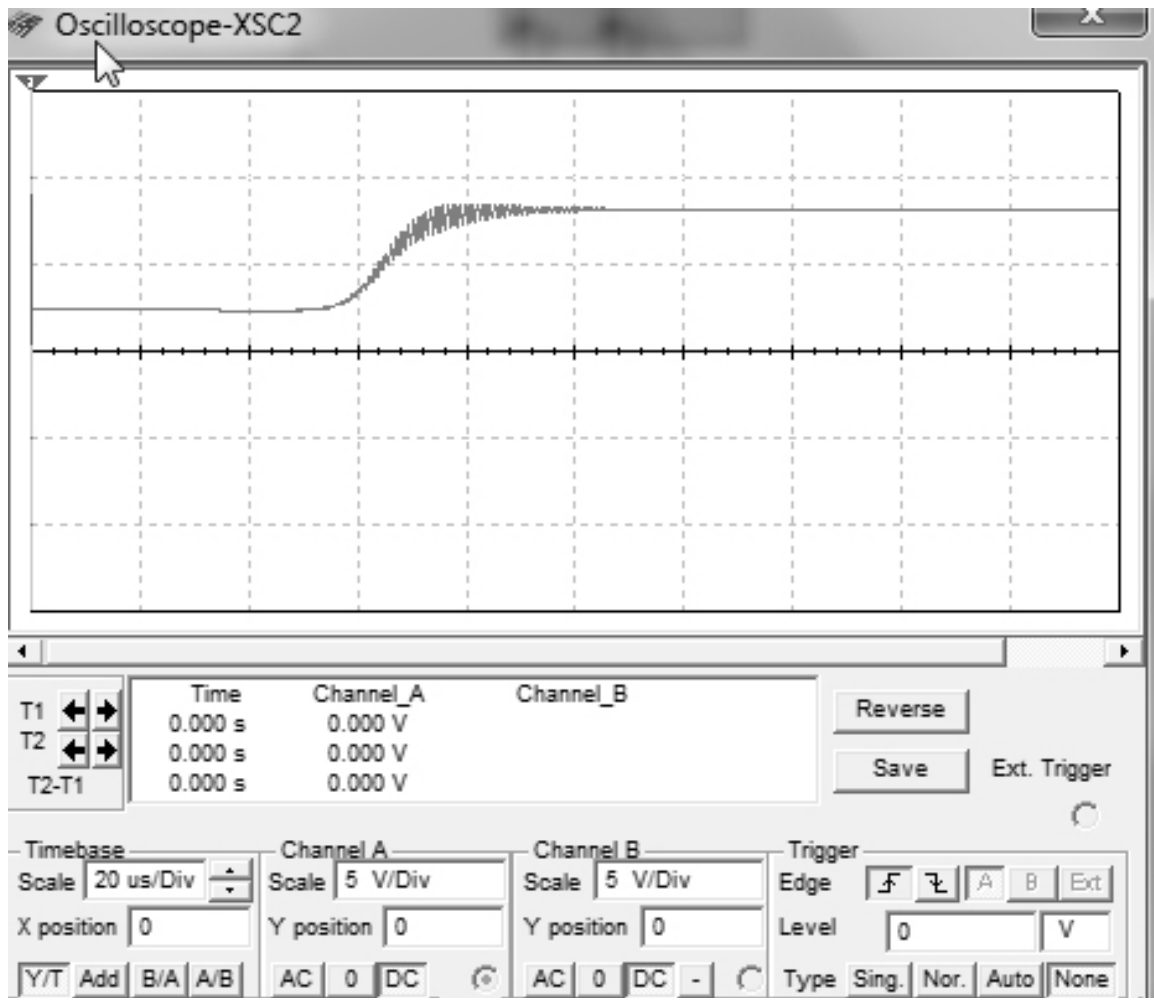

Figure 6: Output waveform for the circuit in figure. 


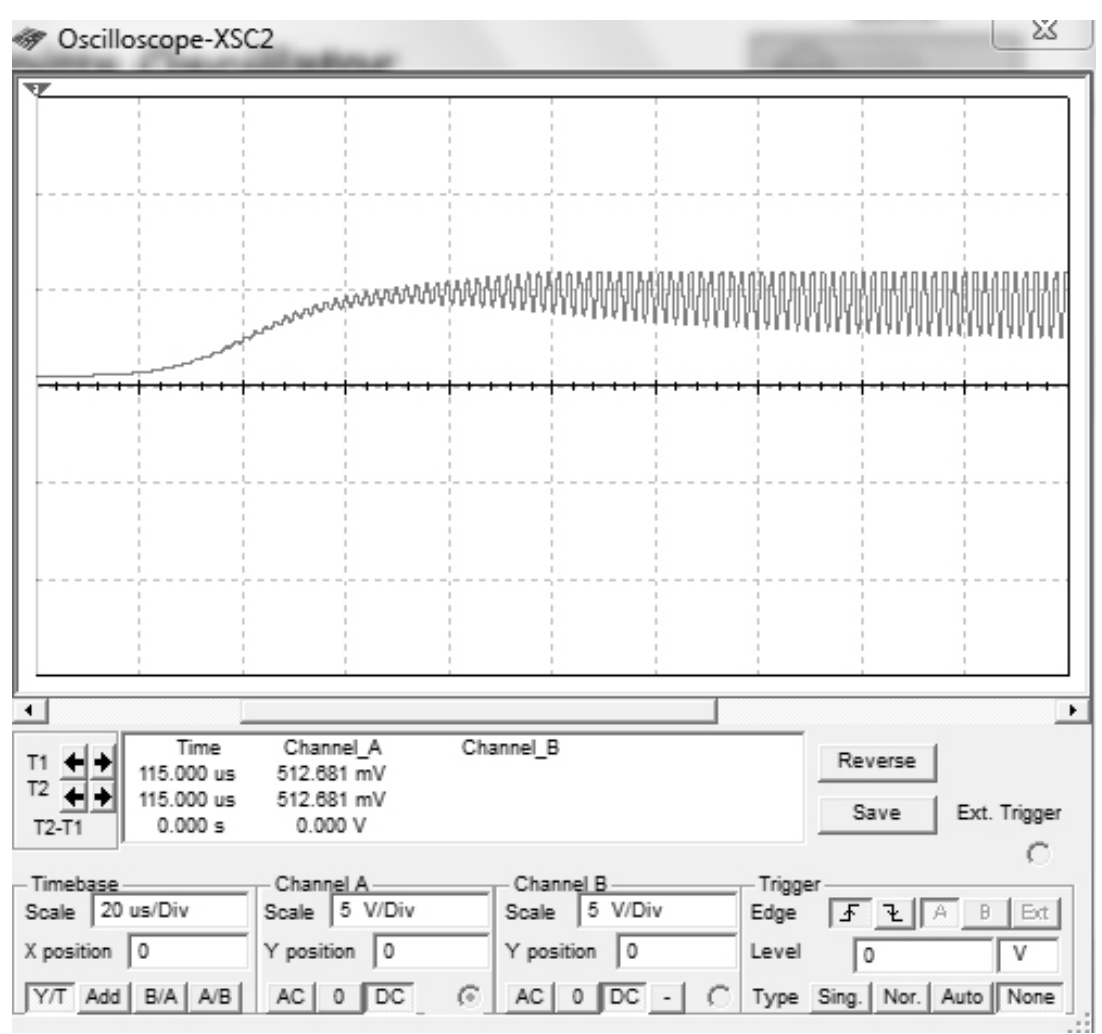

Figure 7: Output waveform for the circuit in figure when the capacitance had been decreased to $60 \mathrm{nF}$ each.'

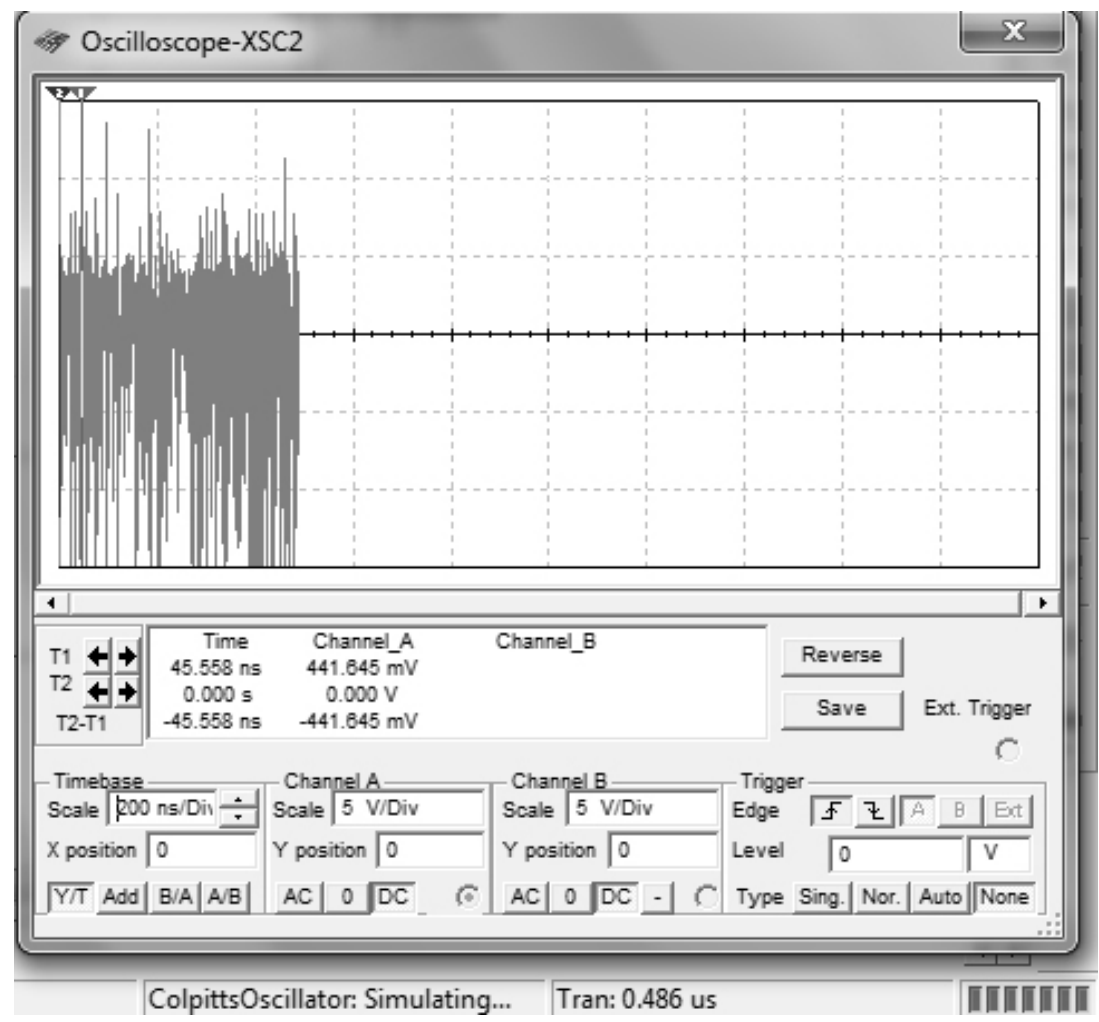

Figure 8: Output waveform for the circuit in figure when the inductance of the coil had been decreased to $2 \mathrm{pH}$. 


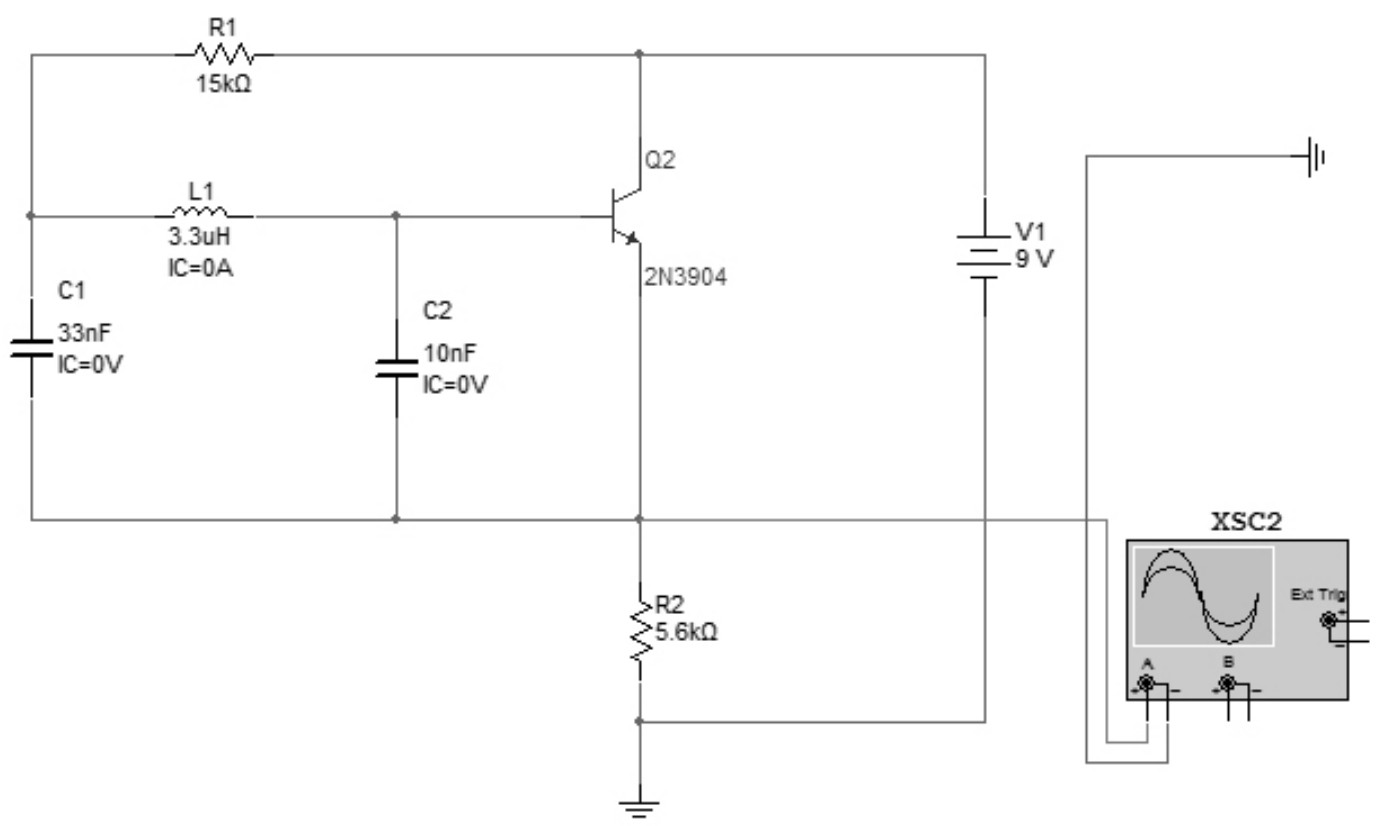

Figure 9: Colpitts Oscillator schematic diagram with a new transistor entity in the simulator design window.

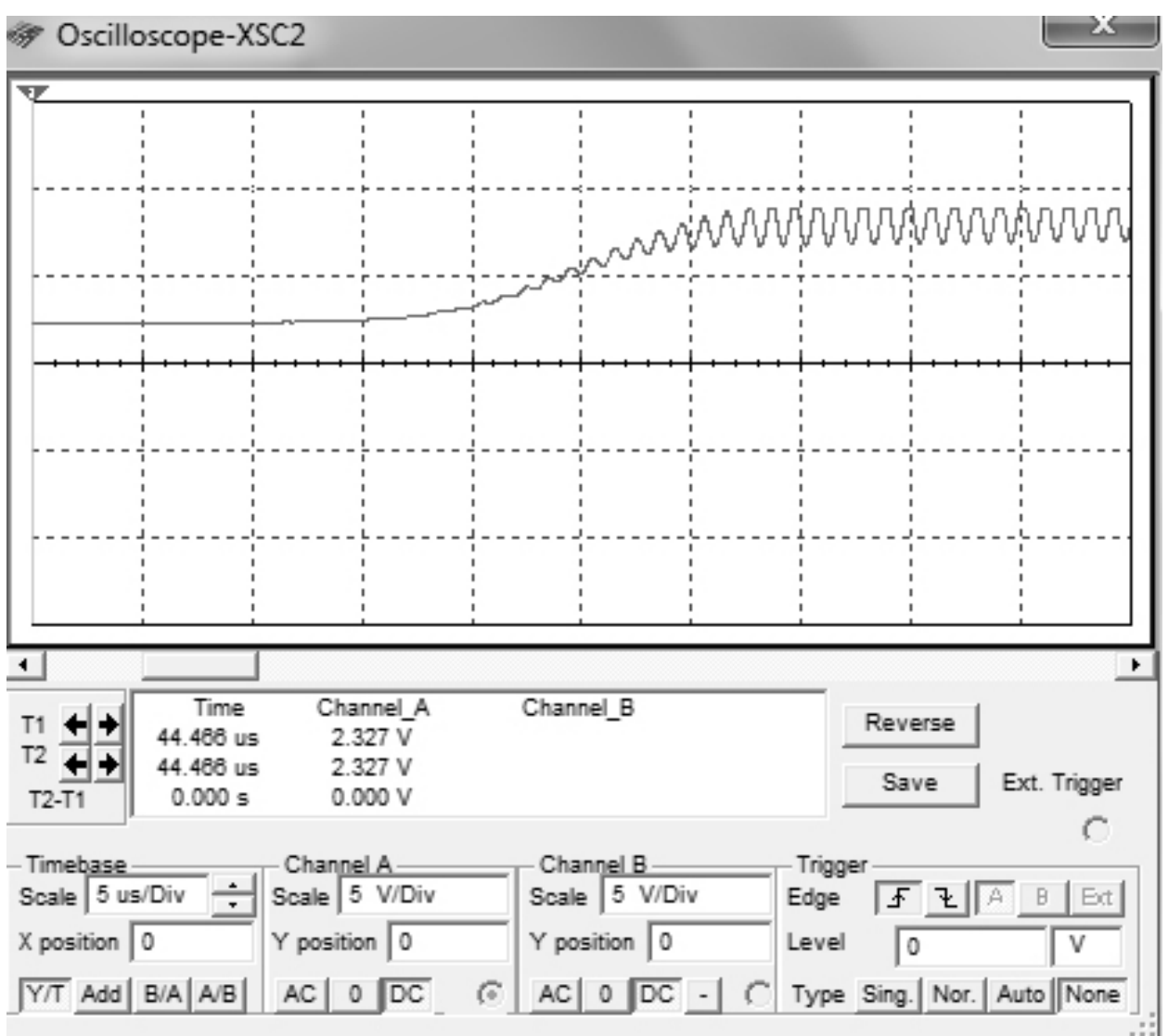

Figure 10: Output waveform for the circuit in figure for tran $0.486 \mathrm{~ms}$ runtime 


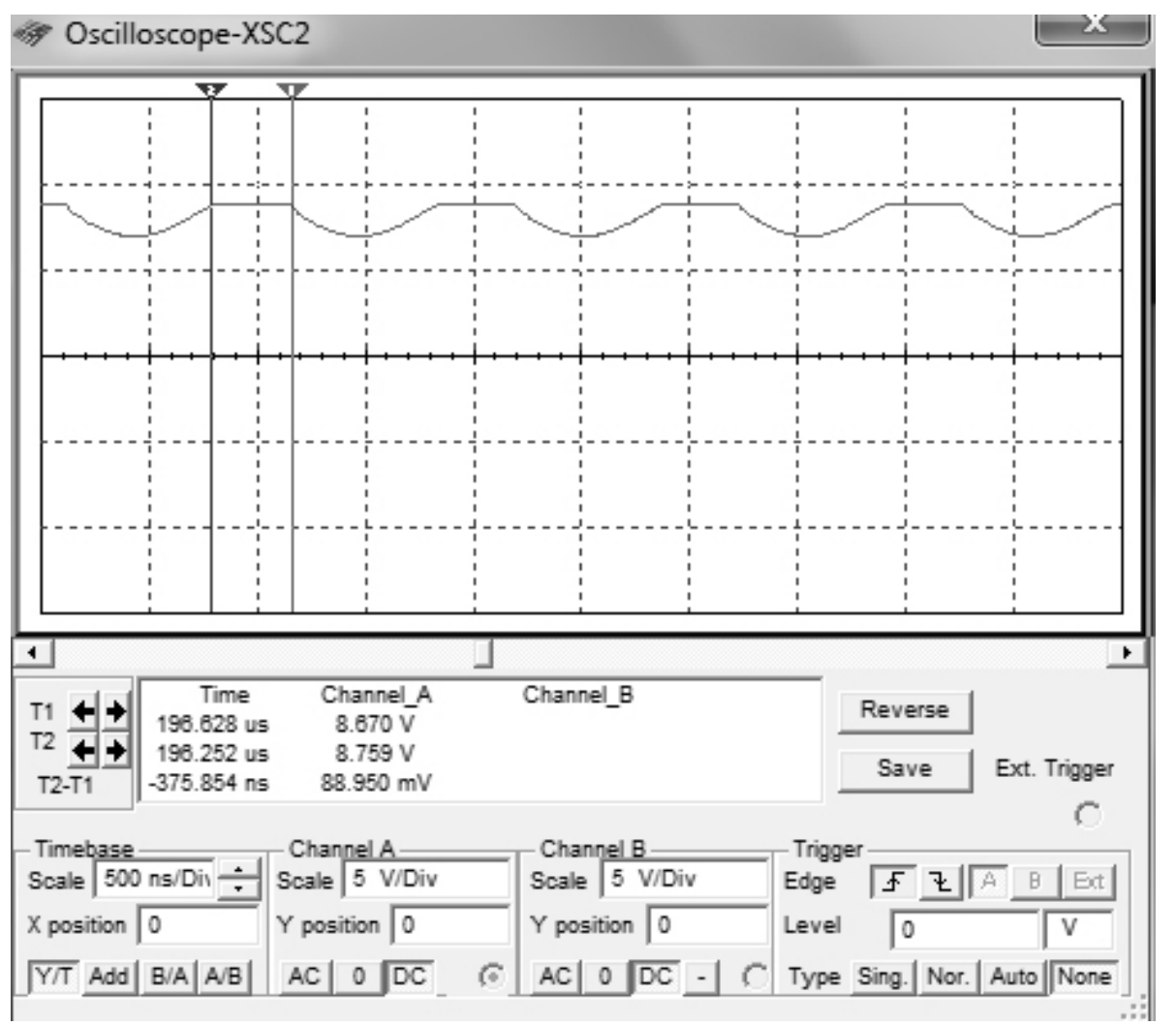

Figure 11: Output waveform for the circuit in figure for pulse flat-top duration in the same runtime.

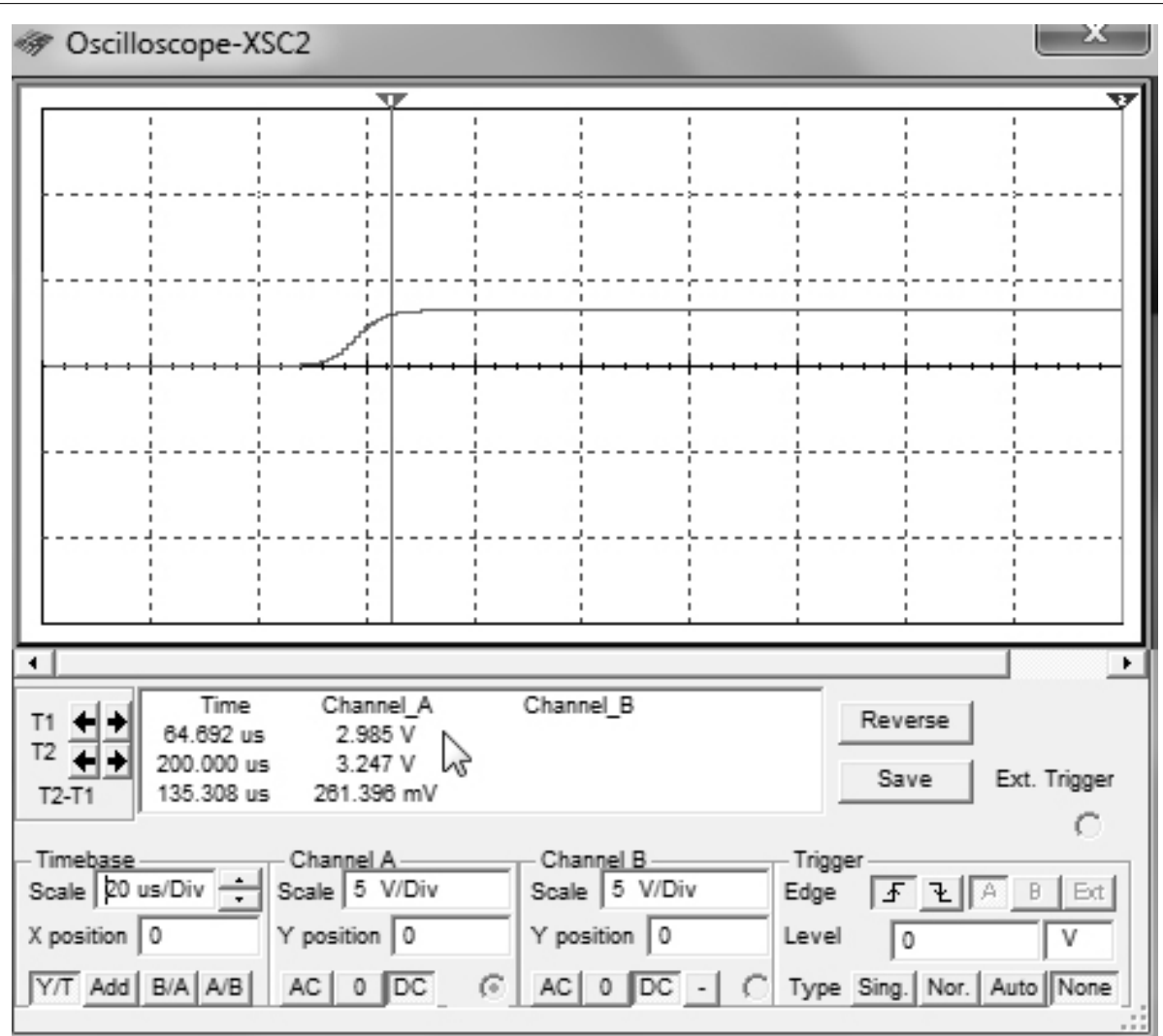

Figure 12: Output waveform for the circuit in figure for resistance value altered to 68 ohms. 


\section{Conclusion}

As is the scenario with any other oscillator design, the amplification (positive or negative) of the active component must be relatively higher than the attenuation shown by the capacitive voltage divider implemented, to obtain a stable functionality or in other words, a smoother sine-wave output. Consequently, a Colpitts oscillator utilized as a variable frequency oscillator (VFO) shows at its best operation when a variant inductance is utilized for tuning the circuit, contrary to the case of tuning one of the two capacitors. In the case, tuning by means of a variable capacitor is required, it must be done by means of a third capacitive entity connected in parallel combination to the inductor coil (or in series). The amount of feedback depends on the values of capacitive entities with the smaller the values of capacitance the more will be the obtained feedback $[3,9]$. The same is optimally adjusted to attain undamped oscillations. In the same scenario, the relative difference or the ratio of two capacitive entities is a big determinant since the two are ganged together. The amplification of the active component must be marginally larger than the attenuation seen due to the voltage divider (capacitive combination), to obtain the most stable operation possible.

\section{References}

1. Razavi B (2001) Design of Analog CMOS Integrated Circuits. McGraw-Hill.

2. Blanchard J (1941) The History of Electrical Resonance. Bell System Technical Journal, USA.

3. Gottlieb I (1997) Practical Oscillator Handbook, Elsevier, USA.

4. Huurdeman AA (2003) The worldwide history of telecommunications, WileyIEEE, USA.

5. Rohde UL, Poddar A, Bock G (2005) The Design of Modern Microwave Oscillators for Wireless Applications: Theory and Optimization, John Wiley \& Sons.

6. Rohde UL, Rudolph M (2012) RF / Microwave Circuit Design for Wireless Applications (2ndedn) John Wiley \& Sons.

7. http://www.howstuffworks.com/oscillator.htm

8. Sarkar S, Sarkar S, Sarkar BC (2013) Nonlinear Dynamics of a BJT Based Colpitts Oscillator with Tunable Bias Current. IJEAT.

9. http://www.ittc.ku.edu/ jstiles/622/handouts/Oscillators $\% 20 \mathrm{~A} \% 20 \mathrm{Brief} \% 20$ History.pdf 